\title{
Flow effects on the freeze-out phase-space density in heavy-ion collisions
}

\author{
Boris Tomášik \\ Department of Physics, University of Virginia, P.O. Box 400714, Charlottesville, Virginia 22904-4714 \\ and CERN, Theory Division, CH-1211 Geneva, Switzerland \\ Ulrich Heinz \\ Department of Physics, The Ohio State University, 174 West 18th Avenue, Columbus, Ohio 43210
}

(Received 21 August 2001; published 8 February 2002)

\begin{abstract}
The strong longitudinal expansion of the reaction zone formed in relativistic heavy-ion collisions is found to significantly reduce the spatially averaged pion phase-space density, compared to naive estimates based on thermal distributions. This has important implications for data interpretation and leads to larger values for the extracted pion chemical potential at kinetic freeze-out.
\end{abstract}

DOI: 10.1103/PhysRevC.65.031902

PACS number(s): 25.75.Ld

The phase-space density of mesons produced in ultrarelativistic heavy-ion collisions is an interesting quantity. Its spatial average at freeze-out (symbolized by $t_{\mathrm{f}}$ ),

$$
\langle f\rangle(\boldsymbol{p})=\frac{\int d^{3} x f^{2}\left(t>t_{\mathrm{f}}, \boldsymbol{x}, \boldsymbol{p}\right)}{\int d^{3} x f\left(t>t_{\mathrm{f}}, \boldsymbol{x}, \boldsymbol{p}\right)},
$$

can be measured by combining the single-particle momentum spectrum with the "homogeneity volume" extracted from Bose-Einstein correlation measurements [1]. Bertsch's original formula [1] was refined in [2] to exclude contributions from long-lived resonances decaying far outside the collision fireball, and a relativistically covariant derivation was given in $[3,4]$. With these improvements Bertsch's formula reads

$$
\langle f\rangle(\boldsymbol{p})=\frac{\sqrt{\lambda(\boldsymbol{p})} d n /\left(d y p_{\perp} d p_{\perp} d \boldsymbol{\phi}\right)}{\left(E_{p} / \pi^{3 / 2}\right) R_{s}(\boldsymbol{p}) \sqrt{R_{o}^{2}(\boldsymbol{p}) R_{l}^{2}(\boldsymbol{p})-R_{o l}^{4}(\boldsymbol{p})}} .
$$

Here $\lambda$ is the intercept at vanishing relative momentum of the Bose-Einstein correlation function, and the factor $\sqrt{\lambda(p)}$ corrects for contributions from decays of long-lived resonances in accord with the core-halo model [5]. The numerator is the (thus corrected) invariant momentum spectrum, and the denominator contains the homogeneity volume calculated from the Hanbury Brown-Twiss (HBT) radii extracted from the Bose-Einstein correlation function [4].

The strongest motivation for measuring the phase-space density of pions at freeze-out (i.e., on the last-scattering surface) comes from the search for multiboson symmetrization effects [6]. Analyses of the hadron yields, spectra, and twoparticle correlations from $\mathrm{Pb}+\mathrm{Pb}$ collisions at the CERN SPS [7] and $\mathrm{Au}+\mathrm{Au}$ collisions at RHIC [8] indicate that freeze-out occurs in two stages: chemical freeze-out, where the particle yields decouple, happens first, reflecting a temperature of around $170 \mathrm{MeV}$, while kinetic or thermal freezeout takes place much later, at temperatures around 100-120 $\mathrm{MeV}$. Between these two points the system cools adiabatically at constant particle numbers which requires the buildup of positive chemical potentials [9]. It has been speculated that for pions, with only $139 \mathrm{MeV}$ rest mass, this chemical potential could approach the Bose condensation limit $\mu_{\pi}$ $=m_{\pi}$. If this were true, measurements of the above type should exhibit large values of $\langle f\rangle(\boldsymbol{p})$ at small $\boldsymbol{p}$. Large phasespace densities also generate significant multiboson symmetrization effects on the pion spectra [10] and Bose-Einstein correlation functions (see [11] and references therein), which lead to a reduction of the homogeneity volume extracted from the standard HBT correlation analysis [4]. This should cause an additional enhancement of $\langle f\rangle(\boldsymbol{p})$ as determined by Bertsch's formula (2), rendering it at the same time unreliable as an estimator of the real average phase-space density.

Previous analyses of heavy-ion data at the AGS [2] and SPS [12,3] concluded that there was no evidence for unusually large pion freeze-out phase-space densities at these collision energies. This conclusion was based on a comparison of $\langle f\rangle(\boldsymbol{p})$ from Eq. (2) with a Bose-Einstein distribution, yielding rough agreement when inserting a kinetic freeze-out temperature of around 100-120 MeV (as extracted by other methods [7]) and a small or vanishing pion chemical potential. In some cases $[3,13]$ a comparison was made with a transversely boosted Bose-Einstein distribution, in order to account for transverse flow effects. We here point out that all these comparisons may have been misleading since they neglected a strong reduction effect on the spatial averaging originating from the longitudinal expansion of the source. We show that for longitudinally expanding sources the spatially averaged phase-space density is smaller than its thermal value in the local rest frame, and that accounting for this effect is likely to considerably increase the extracted value of the pion chemical potential at kinetic freeze-out. In this Rapid Communication we concentrate on a theoretical exposition of the basic mechanism and leave a (re)analysis of existing data for later.

For a qualitative understanding of the effect under discussion, let us for simplicity assume a fireball of constant matter density. We fix the momentum and focus on the averaging over position space. If the (thermalized) fireball expands, different parts moving with different velocities contribute to the production of particles with fixed momentum $\boldsymbol{p}$ with different rates, given by Bose-Einstein distributions boosted by the 
local flow velocity. The phase-space density at momentum $\boldsymbol{p}$ then becomes a function of position space. For an average of type (1), where the function is weighted with itself, the result is always smaller than the maximum of the function (here corresponding to zero flow velocity). In general the $\boldsymbol{p}$ dependence of the spatial average will no longer be of BoseEinstein form. (Obviously, there is no position dependence of the emission rate in a nonexpanding source for which the spatial average would thus coincide with the underlying distribution.)

We illustrate this effect quantitatively within a specific model for the expanding source. We will assume a thermalized and longitudinally boost-invariant fireball. This simple model is expected to provide a good description of pions emitted near midrapidity at RHIC and LHC energies. We show that the calculated $p_{\perp}$ dependence of the spatially averaged phase-space density is flatter than a Bose-Einstein distribution with the assumed freeze-out temperature. The major contribution to this flattening arises from the longitudinal expansion which strongly reduces $\langle f\rangle(\boldsymbol{p})$ relative to the corresponding static Bose-Einstein distribution. Transverse flow introduces a weaker additional flattening by shifting weight from low to high transverse momenta $p_{\perp}$.

The phase-space density $f(t, \boldsymbol{x}, \boldsymbol{p})$ is related to the source emission function $S(x, \boldsymbol{p})[4]$ by

$$
f(t, \boldsymbol{x}, \boldsymbol{p})=\frac{(2 \pi)^{3}}{E_{p}} \int_{-\infty}^{t} d t^{\prime} S\left(t^{\prime}, \boldsymbol{x}+\boldsymbol{v}\left(t^{\prime}-t\right), \boldsymbol{p}\right),
$$

where $\boldsymbol{v}=\boldsymbol{p} / E_{p}$ is the velocity corresponding to the momentum $\boldsymbol{p}$. The prefactor $(2 \pi)^{3} / E_{p}$ ensures the standard normalization

$$
\bar{N}=\int \frac{d^{3} x d^{3} p}{(2 \pi)^{3}} f(t, \boldsymbol{x}, \boldsymbol{p})=\int \frac{d^{3} p}{E_{p}} d^{4} x S(x, \boldsymbol{p}) .
$$

We take $\bar{N}$ as the average number of "directly emitted" pions, including those from the decays of short-lived resonances but excluding those from resonances with long lifetimes [2,12]. For simplicity, we follow Refs. [14,15] and assume that the change in shape and normalization of the thermal pion distribution at freeze-out caused by adding pions from short-lived resonance decays can be effectively absorbed by giving the pions a positive chemical potential. Although this cannot replace a full resonance decay calculation using the proper decay kinematics [16], it qualitatively reproduces the decay-induced low- $p_{\perp}$ enhancement of the measured pion spectra [14-16].

Equation (1) is brought into the form (2) by following the steps outlined in Sec. 3.4 of Ref. [4]. In order to include all "directly emitted" pions, the time $t$ on the right-hand side of Eq. (1) must be later than the time at which the last pion was emitted. Due to Liouville's theorem, the average phase-space density (1) remains constant after completion of the freezeout process. This allows us [3] to replace in Eq. (1) the spatial integrals at constant time $t$ by integrals over the freeze-out hypersurface $\Sigma_{\mathrm{f}}(x)$ describing the last pion scattering or production points:

$$
\langle f\rangle(\boldsymbol{p})=\frac{\int_{\Sigma_{\mathrm{f}}} p \cdot d^{3} \sigma(x) f^{2}(x, \boldsymbol{p})}{\int_{\Sigma_{\mathrm{f}}} p \cdot d^{3} \sigma(x) f(x, \boldsymbol{p})} .
$$

If $\Sigma_{\mathrm{f}}(x)$ is parametrized by an $x$ dependent freeze-out time $t_{\mathrm{f}}(\boldsymbol{x})$, then

$$
\begin{aligned}
& \int_{\Sigma_{\mathrm{f}}} p \cdot d^{3} \sigma(x) f^{n}(t, \boldsymbol{x}, \boldsymbol{p}) \\
& \quad=E_{p} \int d^{3} x\left(1-\boldsymbol{v} \cdot \nabla_{x} t_{\mathrm{f}}(\boldsymbol{x})\right) f^{n}\left(t_{\mathrm{f}}(\boldsymbol{x}), \boldsymbol{x}, \boldsymbol{p}\right) .
\end{aligned}
$$

For the model emission function we take [17]

$$
S(x, p)=\frac{m_{\perp} \cosh (y-\eta)}{(2 \pi)^{3}} \frac{\delta\left(\tau-\tau_{\mathrm{f}}\right)}{\exp \left[\frac{p \cdot u(x)-\mu(r)}{T}\right]-1}
$$

where

$p \cdot u(x)=m_{\perp} \cosh (y-\eta) \cosh \zeta(r)-p_{\perp} \cos (\phi-\varphi) \sinh \zeta(r)$.

We use longitudinal proper time $\tau=\sqrt{t^{2}-z^{2}}$, space-time rapidity $\eta=\frac{1}{2} \ln [(t+z) /(t-z)]$, and transverse polar coordinates $(r, \varphi)$ to parametrize $x$. The pion momentum is $p$ $=\left(m_{\perp} \cosh y, p_{\perp} \cos \phi, p_{\perp} \sin \phi, m_{\perp} \sinh y\right)$. Equation (7) describes a longitudinally infinite source with boost-invariant longitudinal expansion and sharp freeze-out at proper time $\tau_{\mathrm{f}}$. Transverse expansion is parametrized by the transverse flow rapidity $\zeta(r)$, to be specified below. The $r$-dependent chemical potential $\mu(r)$ allows us to discuss different transverse density profiles of the source at freeze-out. While probably too simple for a quantitative comparison with data, this model is technically convenient and allows to investigate the effects of longitudinal and transverse flow as well as the influence of the chemical potential on the average phasespace density $\langle f\rangle(\boldsymbol{p})$.

We now evaluate Eq. (5) for midrapidity pions $(y=0)$. We can rotate the coordinate frame such that $\phi=0$. From Eq. (3) we obtain for the phase-space density immediately after freeze-out

$$
f\left(t_{\mathrm{f}}(\boldsymbol{x}), \boldsymbol{x}, \boldsymbol{p}\right)=\left[\exp \left(\frac{p \cdot u\left(x_{\mathrm{f}}\right)-\mu(r)}{T}\right)-1\right]^{-1},
$$

with $x_{\mathrm{f}}=\left(t_{\mathrm{f}}(\boldsymbol{x}), \boldsymbol{x}\right)$. For the integration in Eq. (6) we use the freeze-out surface at $\tau=\tau_{\mathrm{f}}$ with integration measure

$$
\left(1-\boldsymbol{v} \cdot \nabla_{x} t_{\mathrm{f}}(\boldsymbol{x})\right) d^{3} x=\tau_{\mathrm{f}} \cosh \eta d \eta r d r d \varphi .
$$

To obtain $\langle f\rangle(\boldsymbol{p})$ from Eq. (5) we need $\left(E_{p}=m_{\perp}\right.$ for $\left.y=0\right)$

$$
A_{n}\left(p_{\perp}\right)=m_{\perp} \tau_{\mathrm{f}} \int \frac{\cosh \eta d \eta r d r d \varphi}{\left[\exp \left(\frac{p \cdot u\left(x_{\mathrm{f}}\right)-\mu(r)}{T}\right)-1\right]^{n}}
$$


for $n=1,2$. Note that $A_{1}\left(p_{\perp}\right)$ is, up to a factor $(2 \pi)^{-3}$, the invariant momentum spectrum $E_{p}\left(d N / d^{3} p\right)$.

We evaluate $A_{n}$ by expanding the Bose distribution as $\left(e^{x}-1\right)^{-1}=\sum_{k=1}^{\infty} e^{-k x}$. This reduces Eq. (10) to a sum of exponential integrals. We now integrate over $\eta$ and $\varphi$ in the standard way [18], obtaining Bessel functions $K_{1}$ and $I_{0}$, respectively. Only the radial integration must be done numerically. With the shorthands

$$
\begin{aligned}
& \alpha_{\perp}(r)=p_{\perp} \sinh \zeta(r) / T \\
& \beta_{\perp}(r)=m_{\perp} \cosh \zeta(r) / T
\end{aligned}
$$

we find

$$
\begin{gathered}
\langle f\rangle\left(p_{\perp}\right)=\frac{A_{2}\left(p_{\perp}\right)}{A_{1}\left(p_{\perp}\right)}, \\
A_{1}\left(p_{\perp}\right)=\sum_{k=1}^{\infty} \widetilde{A}_{k}\left(p_{\perp}\right), \\
A_{2}\left(p_{\perp}\right)=\sum_{k=2}^{\infty}(k-1) \tilde{A}_{k}\left(p_{\perp}\right), \\
\widetilde{A}_{k}\left(p_{\perp}\right)=4 \pi m_{\perp} \tau_{\mathrm{f}} \int_{0}^{\infty} r d r e^{k[\mu(r) / T]} \\
\times I_{0}\left(k \alpha_{\perp}(r)\right) K_{1}\left(k \beta_{\perp}(r)\right) .
\end{gathered}
$$

We will consider two models for the transverse density profile: a boxlike density

$$
\text { [box] } \mu(r)= \begin{cases}\mu_{B} & \text { for } r \leqslant R_{\text {box }} \\ -\infty & \text { otherwise, }\end{cases}
$$

and a Gaussian profile

$$
\text { [Gauss] } \mu(r)=\mu_{G}-T \frac{r^{2}}{2 R_{\text {Gauss }}^{2}} \text {. }
$$

Here $\mu_{B}$ and $\mu_{G}$ are the chemical potential values in the center of the fireball. For the Gaussian profile, $\mu_{G}$ will be larger than the global (averaged) chemical potential used in chemical analyses of particle abundances [19].

As a warm-up, let us consider the case without transverse flow, $\zeta(r)=0$, in the Boltzmann approximation (i.e., keeping only the first term in the sums over $k$ ). Then

$$
\left\langle f_{\zeta=0}^{\text {Boltz }}\right\rangle\left(p_{\perp}\right)=\bar{\lambda}_{\pi} \frac{K_{1}\left(2 \frac{m_{\perp}}{T}\right)}{K_{1}\left(\frac{m_{\perp}}{T}\right)}
$$

where $\bar{\lambda}_{\pi}$ is the spatially averaged pion fugacity

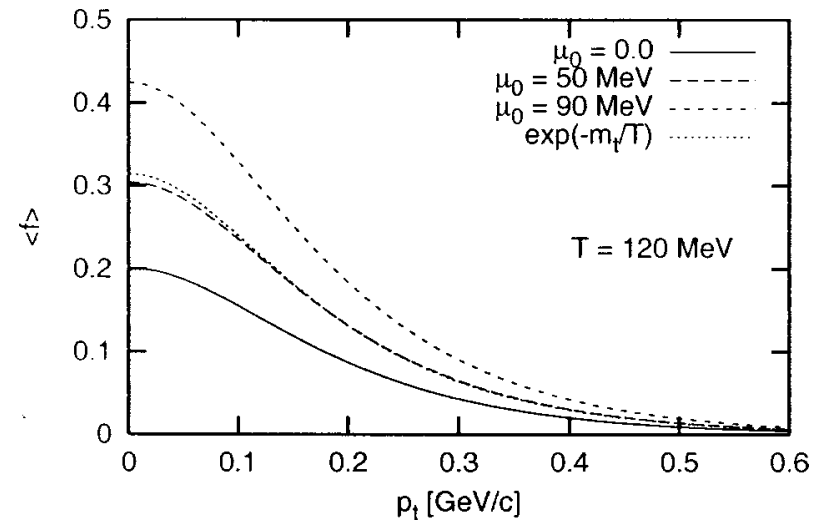

FIG. 1. Average phase-space density $\langle f\rangle\left(p_{\perp}\right)$ at rapidity $y=0$ for pions from a longitudinally expanding fireball with Boltzmanndistributed particle momenta, for a temperature of $120 \mathrm{MeV}$ and three representative values for the pion chemical potential.

$$
\bar{\lambda}_{\pi}=\left\langle e^{\mu / T}\right\rangle \equiv \frac{\int r d r e^{2 \mu(r) / T}}{\int r d r e^{\mu(r) / T}},
$$

the average being taken with the transverse matter density $\rho(r) \sim e^{\mu(r) / T}$. A boxlike density gives $\bar{\lambda}_{\pi}=e^{\mu_{B} / T}$ whereas a Gaussian profile leads to $\bar{\lambda}_{\pi}=\frac{1}{2} e^{\mu_{G} / T}$.

In Fig. 1 we show the result (14) for $T=120 \mathrm{MeV}$ and three values for the average pion fugacity $\bar{\lambda}_{\pi}$. According to Eq. (14) the curves do not depend on the transverse density profile. The calculation in Fig. 1 was done with a boxlike density profile for which $\mu_{B}$ can be considered as the global chemical potential. The same curves would be obtained in the Gaussian model by adjusting $\mu_{G}$ such that $e^{\mu_{G} / T}$ $=2 e^{\mu_{B} / T}$ [leading to a twice larger fugacity $\lambda_{\pi}(r=0)$ $=2 \bar{\lambda}_{\pi}$ in the fireball center].

The dotted line in Fig. 1 is shown for reference and represents a Boltzmann distribution with vanishing pion chemical potential for the same temperature as used in the calculations (i.e., the original thermal pion momentum distribution in the local rest frame). Comparing it with the solid curve one sees that, even without transverse flow, at low $p_{\perp}$ the spatially averaged phase-space density of the longitudinally expanding source is about $35 \%$ lower than its value in the local rest frame. In order to obtain an $\langle f\rangle\left(p_{\perp}\right)$ which looks like a Boltzmann distribution with vanishing pion chemical potential, we need in fact an average pion fugacity $\bar{\lambda}_{\pi}$ $\approx 1.55$. For practical purposes this interpretation is better presented the other way around: if the curve corresponding to $\bar{\lambda}_{\pi} \approx 1.55$ (or $\mu_{B}=50 \mathrm{MeV}$ at $T=120 \mathrm{MeV}$ ) was measured and then fitted by a Boltzmann distribution with $T$ $=120 \mathrm{MeV}$, one would erroneously conclude $\mu_{B}=0$.

For large $m_{\perp}, K_{1}\left(2 m_{\perp} / T\right) / K_{1}\left(m_{\perp} / T\right) \approx e^{-m_{\perp} / T}$, and this type of error no longer arises. Unfortunately, this requires for pions much larger values of $p_{\perp}$ than presently experimentally accessible. The reason for the strong reduction at low $p_{\perp}$, resulting from taking the spatial average, is that this 
average extends over a large longitudinal homogeneity length, and that for a longitudinally expanding source $f\left(x ; y=0, p_{\perp}\right)$ agrees with the static Boltzmann distribution $\exp \left(-m_{\perp} / T\right)$ only at the point $\eta=0$, being smaller everywhere else by a factor $\exp \left[-m_{\perp}(\cosh \eta-1)\right]$. For large $p_{\perp}$ the longitudinal homogeneity length decreases [4] and the reduction due to spatial averaging becomes less severe.

We now discuss the more general case of a source with additional transverse flow, including the full Bose-Einstein distribution by summing over all $k$ in Eq. (12). The transverse flow profile $\zeta(r)$ influences the radial averaging of the factors containing the pion chemical potential, and it is no longer possible as in Eq. (14) to factorize an effective average pion fugacity $\bar{\lambda}_{\pi}$ from the result for $\langle f\rangle\left(p_{\perp}\right)$. For the transverse flow rapidity we take a linear profile with slope $\eta_{t}$,

$$
\zeta(r)=\eta_{t} \frac{r}{r_{\mathrm{rms}}}
$$

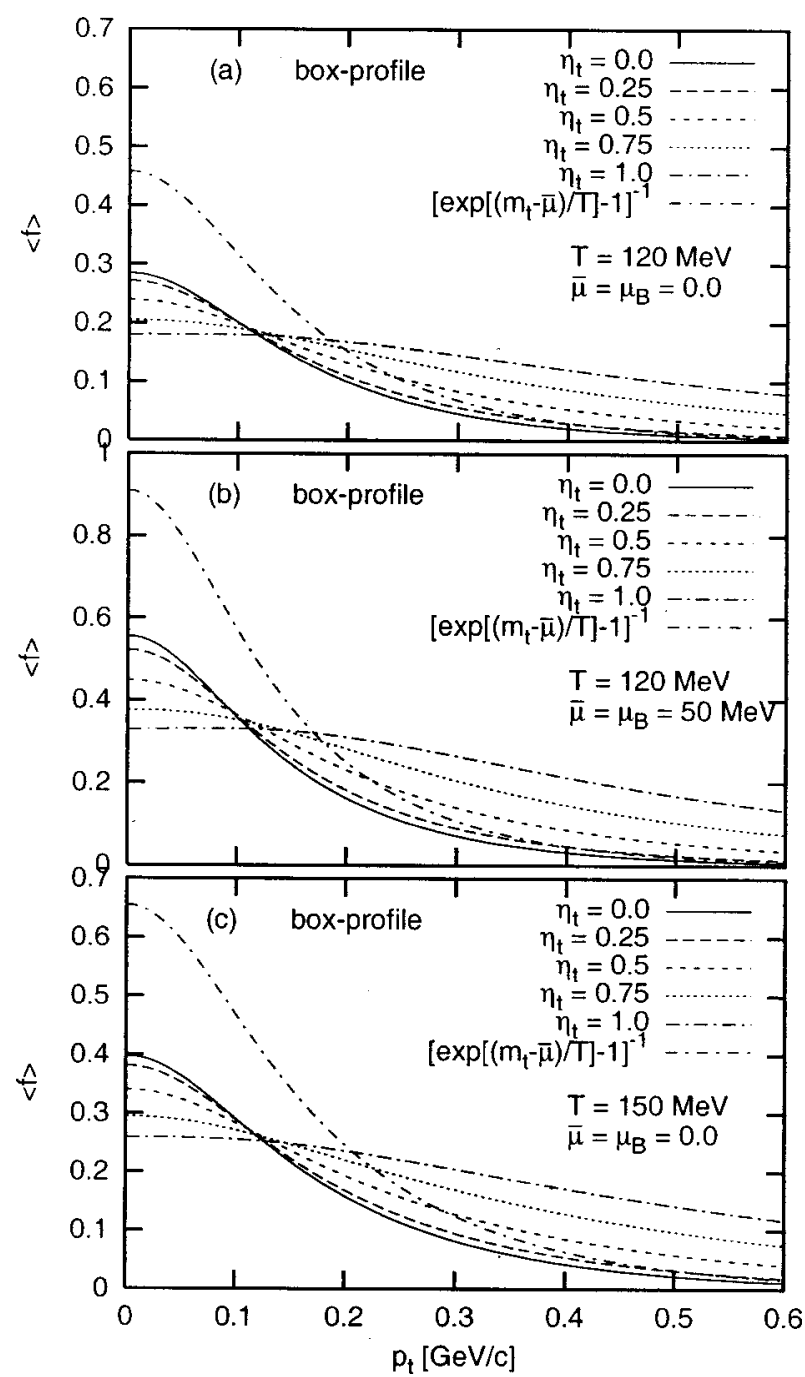

FIG. 2. Average phase-space density $\langle f\rangle$ for pions with $y=0$ from a longitudinally and transversally expanding fireball with Bose-Einstein distributed momenta of temperature $120 \mathrm{MeV}$. The transverse flow is controlled by $\eta_{t}$. The transverse density profile has the shape of a box.

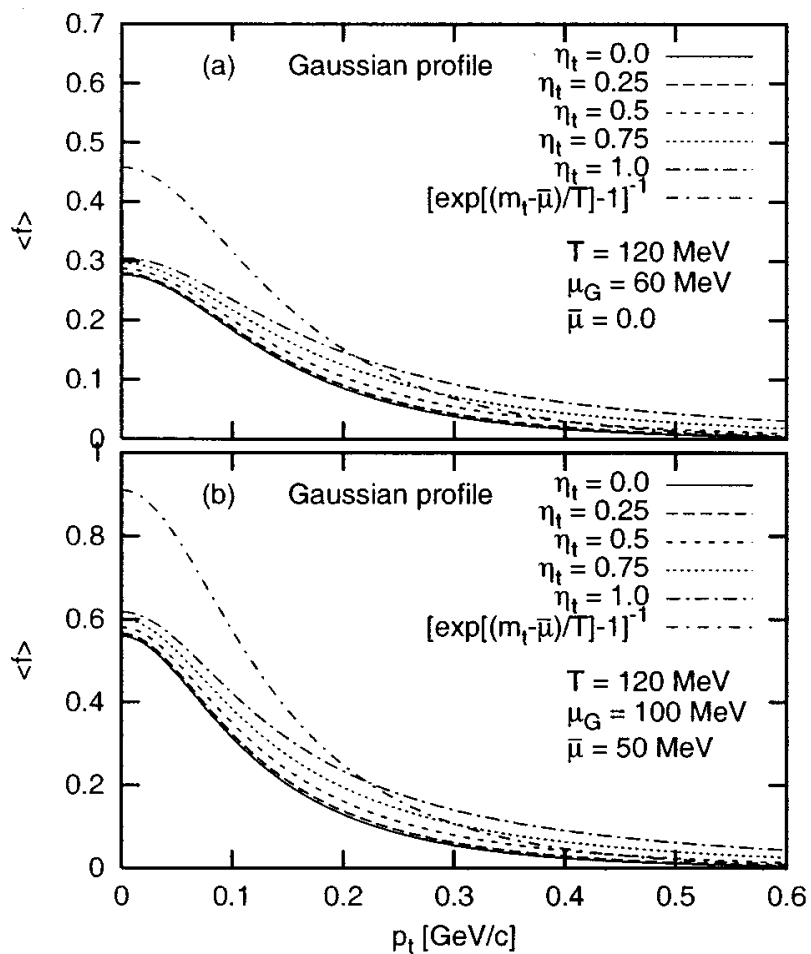

FIG. 3. Same as Fig. 2 but for a Gaussian transverse density profile.

where $r_{\text {rms }}=R_{\text {box }} / \sqrt{2}$ and $r_{\text {rms }}=\sqrt{2} R_{\text {Gauss }}$, respectively, are the rms radii corresponding to the two different transverse density profiles. Thus $\eta_{t}=\zeta_{\text {rms }}$ can be interpreted as the average (rms) transverse flow rapidity.

Figures 2 and 3 show that the longitudinal flow is again responsible for most of the suppression of $\langle f\rangle(\boldsymbol{p})$ compared to the local rest frame distribution. Transverse flow tends to flatten the $p_{\perp}$ dependence of $\langle f\rangle$ rather than to suppress $\langle f\rangle$. This effect stems from enhanced contributions at higher transverse momenta from outward-moving fluid cells; this is the well-known "blue-shift" of the transverse momentum spectrum. The details of this flattening depend on the particular model for the transverse density profile.

The calculated $\langle f\rangle(\boldsymbol{p})$ in Figs. 2 and 3 are compared to Bose-Einstein distributions with an averaged chemical potential $\bar{\mu}$ which takes into account the averaging over the transverse density profile. It is obtained by calculating the "transverse average"

$$
\bar{f}_{\mathrm{tr}}(\boldsymbol{p})=\frac{\int_{0}^{\infty} r d r f_{\mathrm{tr}}^{2}(r, \boldsymbol{p})}{\int_{0}^{\infty} r d r f_{\mathrm{tr}}(r, \boldsymbol{p})}
$$

of the underlying local distribution without flow

$$
f_{\mathrm{tr}}(r, \boldsymbol{p})=\frac{1}{\exp \left[\left(m_{\perp}-\mu(r)\right) / T\right]-1},
$$

where $\mu(r)$ characterizes the chosen transverse density distribution. Then a Bose-Einstein distribution $f_{\mathrm{BE}}$ 
$=1 /\left[\exp \left(\left(m_{\perp}-\bar{\mu}\right) / T\right)-1\right]$ with the same temperature is fitted to $\bar{f}_{\text {tr }}(\boldsymbol{p})$, using $\bar{\mu}$ as a fit parameter to ensure the same normalization $\int f_{\mathrm{BE}}\left(m_{\perp}\right) m_{\perp} d m_{\perp}$. Obviously, for the box profile $\bar{\mu}=\mu_{B}$; for the Gaussian profile, $\bar{\mu}<\mu_{G}$. For the Gaussian profile the Bose fit is reasonable but not perfect since $\bar{f}_{\text {tr }}(\boldsymbol{p})$ is a bit more strongly peaked at small $p_{\perp}$ than for the box profile. However, since $\bar{\mu} / T$ controls the normalization of $f$, i.e., the total pion yield at midrapidity $y=0, \bar{\mu}$ is identical with the global chemical potential extracted from a chemical analysis of midrapidity particle abundances at the thermal freeze-out temperature, and therefore a good reference value.

For the direct comparison of the two transverse density profiles we proceed as follows: we fix $\bar{\mu}$ and, for the Gaussian profile, adjust $\mu_{G}$ accordingly. A comparison of the solid lines in panels (a),(b) of Figs. 2 and 3 shows that in the absence of transverse flow, at the same value of $\bar{\mu}$, the dilution effects on $\langle f\rangle(\boldsymbol{p})$ from longitudinal expansion are almost identical for both density profiles. However, the additional flattening effects from transverse flow are weaker in the Gaussian model than for the box profile.

We have demonstrated that the thermal model of ultrarelativistic heavy-ion collisions does not predict an average phase-space density characterized by a Bose-Einstein distribution function. Comparing data for $\langle f\rangle(\boldsymbol{p})$ extracted from Eq. (2) with such a distribution is likely to severely underestimate the pion chemical potential or temperature. The previously used ad hoc introduction of a naive transverse blueshift factor [3,13], without first properly accounting for the dominant effects from longitudinal expansion, is based on faulty intuition and misleading. Longitudinal expansion strongly reduces the average freeze-out phase-space density at low $p_{\perp}$, whereas transverse flow leads to an additional flattening of $\langle f\rangle\left(p_{\perp}\right)$. At low $p_{\perp}$, where $\langle f\rangle(\boldsymbol{p})$ and thus the danger of distortions due to strong multiboson symmetrization effects [11] are largest, the strong longitudinal expansion of the heavy-ion collision fireball dominates the suppression of the average phase-space density. An extraction of the pion chemical potential should be based on a comparison of the measured values for $\langle f\rangle(\boldsymbol{p})$ with Eqs. (12), or a suitable generalization thereof which more accurately accounts for shortlived resonance decays (which we treated here rather superficially).

We thank John Cramer and Jim Draper for stimulating discussions and valuable comments on a first draft of this paper. The research of U.H. was supported by the U.S. Department of Energy under Contract No. DE-FG0201ER41190.
[1] G.F. Bertsch, Phys. Rev. Lett. 72, 2349 (1994); 77, 789 (1996).

[2] J. Barette et al., E877 Collaboration, Phys. Rev. Lett. 78, 2916 (1997)

[3] B. Tomášik, U.A. Wiedemann, and U. Heinz, nucl-th/9907096.

[4] U.A. Wiedemann and U. Heinz, Phys. Rep. 319, 145 (1999).

[5] T. Csörgö, B. Lörstad, and J. Zimányi, Z. Phys. C 71, 491 (1996).

[6] S. Pratt, Phys. Lett. B 301, 159 (1993); Phys. Rev. C 50, 469 (1994).

[7] For a review see U. Heinz, Nucl. Phys. A685, 414c (2001).

[8] Proceedings of Quark Matter 2001 Stony Brook, New York, 2001 [Nucl. Phys. A698 (2002)].

[9] This is most easily seen in the nonrelativistic Boltzmann limit where the entropy per particle is $s / n=5 / 2+(m-\mu) / T$ : as the temperature $T$ decreases, the chemical potential $\mu$ must increase to maintain constant entropy at constant particle number. For relativistic bosons see H. Bebie, P. Gerber, J.L. Goity, and H. Leutwyler, Nucl. Phys. B378, 95 (1992).

[10] R. Lednický et al., Phys. Rev. C 61, 034901 (2000).

[11] U. Heinz, P. Scotto, and Q.H. Zhang, Ann. Phys. (N.Y.) 288, 325 (2001).

[12] D. Ferenc et al., Phys. Lett. B 457, 347 (1999).

[13] J.G. Cramer, poster at the conference Quark Matter 2001 Stony Brook, New York, 2001.

[14] M. Kataja and P.V. Ruuskanen, Phys. Lett. B 243, 181 (1990).

[15] P. Gerber, H. Leutwyler and J.L. Goity, Phys. Lett. B 246, 513 (1990).

[16] J. Sollfrank, P. Koch, and U. Heinz, Phys. Lett. B 252, 256 (1990).

[17] T. Csörgő and B. Lörstad, Phys. Rev. C 54, 1390 (1996).

[18] U.A. Wiedemann, P. Scotto, and U. Heinz, Phys. Rev. C 53, 918 (1996).

[19] U. Heinz, Nucl. Phys. A661, 140 (1999). 